Oral contraceptives should not be given to patients who have previously suffered pruritus in pregnancy, and special care in the prescribing of them should be taken in countries where intrahepatic cholestatic jaundice of pregnancy is frequent.

In a woman's first pregnancy the differential diagnosis of idiopathic cholestatic jaundice from viral hepatitis and other conditions causing jaundice may be difficult. It might be possible to perform a diagnostic test after delivery. Serial serum-bilirubin, alkaline-phosphatase, and bromsulphalein tests are done before, during, and at the end of a one-cycle course of an oral contraceptive drug in standard dosage. Increasing cholestasis would confirm the diagnosis of intrahepatic cholestatic jaundice of pregnancy, contraindicate further use of this method of contraception, and give warning that subsequent pregnancies might be complicated by intrahepatic cholestatic jaundice. Differential diagnosis must also be made from surgical jaundice. In some cases laparotomy has been undertaken, because of the unusually long duration of jaundice and a preceding history of operation for gallstones. In a recent well-documented case report ${ }^{14}$ the liver was found not to be enlarged, but greatly dilated bile capillaries were visible on its surface. Cholangiography showed a normal appearance, and the amount of bile secreted, the secretion pressure, and its viscosity were found to be within normal limits. The patient subsequently had a normal spontaneous delivery, and the child was healthy. The aetiology of this condition is unknown, but the prognosis is nevertheless excellent. In this patient the jaundice disappeared within three weeks and hepatic histology returned to normal within three months of delivery despite the persistence of jaundice for six months of the pregnancy.

There are certainly many other causes of jaundice during pregnancy, for it has been estimated that it complicates one of every 1,500 gestations. ${ }^{4}$ Viral hepatitis accounts for twofifths of cases, intrahepatic cholestasis for one-fifth, and obstruction of the cominon bile duct for one in 20 . The acute fatty liver of the third trimester of pregnancy is a condition observed particularly in the malnourished patient with pyelonephritis treated with a tetracycline. The liver cells contain multiple fat droplets without significant necrosis or cellular infiltrate. The liver in pregnancy, when demands for protein anabolism are increased, may be more sensitive than in the non-pregnant woman to drugs which depress this function, such as the tetracyclines. The biochemical pattern is of increased serum levels of bilirubin, reduced prothrombin time, azotaemia, hyperuricaemia, and acidosis. Serum values of transaminase and alkaline phosphatase are only moderately

1 Ljunggren, G., Nord. Med., 1956, 55, 373. Caroli, J., Puyo, G., and Rampon, Sem. Hôp. Paris, 1954, 30 ,
1692.

King, M. J., and Kerrins, J. F., New Engl. f. Med., 1963, 268, 1180.

- Haemmerli, U. R., Acta med. scand., 1966, Suppl. 444.

s Sherlock, S., Diseases of the Liver and Biliary System, 3rd ed. 1963. Oxford.

- Eliakim, M., Sadovsky, E., Stein, O., and Shenkar, Y. G., Arch. intern. Med., 1966, 117, 696.

' Adlercreutz, H., Svanborg, A., and Ånberg, A., Amer. F. Med., 1967, 42, 335.

- Mueller, M. N., and Kappas, A., f. clin. Invest., 1964, 43, 1905.

- Orellana-Alcalde, J. M., and Dominguez, J. P., Lancet, 1966, 2, 1278. ${ }^{10}$ Kleiner, G. J., Kresch, L., and Arias, I. M., New Engl. F. Med., 1965,

"Larsson-Cohn, U., and Stenram, U., Acta med. scand., 1967, 181, 257. 12 Holzbach, R. T., and Sanders, J. H., f. Amer. med. Ass., 1965, 193,

18 Thulin, K. E., and Nermark, J., Brit. med. F., 1966, 1, 584.

14 Adlercreutz, H., Svanborg, A., and Ånberg, A., Amẹ. F. Med., 1967, 42. 341.

is Sheehan, H. L., Amer. F. Obstet. Gynec., 1961, 81, 427. raised, a point of distinction from virus hepatitis. The prognosis of acute fatty liver of pregnancy is poor and there is no clear-cut course of management ; caesarean section has been advocated. 4 Corticosteroids are of no value.

Though tests of liver function in patients with toxaemia of pregnancy may give abnormal results, particularly the alkalinephosphatase and serum-transaminase tests, jaundice is infrequent. It was present in $\mathbf{1 0}$ out of $\mathbf{9 0}$ cases of toxaemia in H. L. Sheehan's necropsy series. ${ }^{1 s}$ It is a grave, terminal sign of haemolysis. Histologically the liver shows periportal deposition of fibrin in the sinusoids, with haemorrhages. Centrizonal necrosis and haemorrhages reflect shock, and an inflammatory reaction is inconspicuous. In eclampsia the damage to the liver is terminal, and toxaemia of pregnancy cannot be regarded as primarily involving the liver. Toxaemia rarely enters into the differential diagnosis of jaundice in pregnancy.

Finally, drug jaundice should not be overlooked. Increased sensitivity to tetracycline has been discussed. Other drugs to be borne in mind include chlorpromazine and halothane, while the sulphonamides, novobiocin, and phenacetin should be considered as possible causes of jaundice in immature or premature babies.

\section{Appointment Systems in General Practice}

The growth of appointment systems is one symptom of the reorganization of general practice which is gathering momentum. Dr. Bruce Cardew estimates in a paper at page 542 of this week's B.M.F. that a third of all N.H.S. practices are now offering a complete appointment service to their patients. Thus 17,000,000 people can now consult their doctors by appointment. That the new pay system is accelerating the movement is suggested by an increase in the first nine months of this year of 2,773 in the number of doctors using the LloydHamol appointments diary, but it is noteworthy that under the Pool system 4,500 doctors had been prepared to finance appointment schemes entirely out of their own pockets.

J. M. Bevan and G. J. Draper, ${ }^{1}$ of the Oxford University Department of Biomathematics, have recently investigated appointment systems. They had three main objectives: to find out the attitudes of doctors and patients to them; to get quantitative estimates of their effect on doctors' work load and patients' waiting time ; and to determine which types of practice would find appointment systems worth while and what were the best ways of organizing them. They formed various theories from interviews with and postal surveys from doctors and patients, and tested them in an experimental study of the records of several practices before and after starting appointment systems. The vast majority of doctors and patients who had used an appointment system were satisfied with it. Nearly all doctors found that it was easy to introduce into their practices, that it evened out the work load throughout the week, and that it decreased the strain of their work and improved its quality. Patients who were elderly or of low intelligence found it difficult at first, especially if they had not been informed beforehand by circulars about its introduction and how to use it, but they liked it once they had got used to it. About half of the doctors who did not have appointment systems at the time of the survey thought them 
desirable but cited overworking of existing staff as the main reason for not starting them. Most of them did not think that reimbursement of part of the cost of ancillary staff would influence this decision, though the marked increase in appointment systems since January suggests that this was a rationalization of their reluctance to take the plunge. Patients showed a similar conservatism. Their satisfaction with the status quo, noted elsewhere by Ann Cartwright, ${ }^{2}$ was reflected by the far less favourable reaction to the idea of appointment systems by patients of doctors who did not have them. Once they had experienced a successful system patients were overwhelmingly in favour of it, probably because in the average practice it halved their waiting time.

In quantitative terms the degree of success of an appointment system can be measured by the proportion of patients actually making appointments and the proportion failing to keep them. These figures varied in the practices studied from $45 \%$ to $96 \%$ and from $0 \%$ to $7 \%$, respectively. Most patients were punctual, and of the $10 \%$ who were not only a third were more than five minutes late. As Dr. Stuart Carne points out at page 544 of this week's B.M.F., an appointment system can also work in a practice with a high proportion of West Indian and West African immigrants, who on the whole are not such good timekeepers as the English and Irish patients.

The success of any system depends to a large extent on the organizing ability of the receptionists and their attitude to patients. They need to be present during all surgery sessions at least, and, on average, staff time must be increased by ten to twelve hours per week per doctor in a partnership and twice as much in a single-handed practice. Success depends also on estimating beforehand the doctor's consultation time-which may differ for each doctor in a partnership - and scheduling appointments accordingly. Doctors must be punctual themselves, because patients who repeatedly have to wait long after their appointment time get disillusioned and stop making appointments.

One of the changes which would help fewer practitioners to care effectively for more patients would be an increase in the ratio of surgery consultations to home visits. The figures from the practices, admittedly small in number, which kept records before and after an appointment system did not show any such increase, though more detailed data and a more rigorous analysis would have been needed to detect any but a very large one. That a consultation probably did acquire greater significance is suggested by the fact that about a quarter of the doctors questioned thought there had been a decrease in trivial consultations and an increase in non-trivial ones. But if an appointment system does not in itself persuade patients to come to the surgery instead of asking for a home visit it is an essential prerequisite for other means of achieving that end: by having a doctor consulting throughout the day as J. S. K. Stevenson ${ }^{3}$ found ; or by receptionists pointing out the convenience of a surgery attendance at a particular time for the patient himself as well as for his doctor, as Andrew Smith ${ }^{4}$ has suggested.

What does emerge quite clearly from the investigation is that it is worth while for every type of practice, except probably a single-handed one, to run a system in which all sessions are by appointment and all patients are encouraged to make an appointment.

\footnotetext{
1 Bevan, J. M., and Draper, G. J., Appointment Systems in General Practice, 1967, London. Oxford University Press, price 12s. 6d. - Cartwright, Ann, Patients and their Doctors, 1967. London.

- Stevenson, J. S. K., Brit. med. Y., 1966, 2, 515.

- Smith, A., ibid., 1967, 2, 369.
}

\section{Reappraisal of the Austin Flint Murmur}

When Alice, in Through the Looking-glass, was trying to reach a particular hill she found that the only way to reach her destination was to turn her back on it and walk in the opposite direction. Something of the same kind seems to be happening to our conception of the Austin Flint murmur.

In Austin Flint's original description of the murmur ${ }^{1}$ he suggested that the likely pathogenesis was that the mitral valve leaflets became closed in early diastole as a result of over-distension of the left ventricle from free aortic incompetence, and that during atrial systole the mitral valve reopened with accompanying vibrations of the anterior cusp, producing a characteristic blubbering murmur. Recently it has been shown that in cases of free aortic incompetence reversal of the left atrioventricular gradient takes place in late diastole, left ventricular exceeding left atrial pressure by $20 \mathrm{~mm}$. Hg or more. ${ }^{2-4}$ E. D. Wigle and C. J. Labrosse in 1965 found that an Austin Flint murmur was present in 9 out of 14 patients who developed sudden severe aortic incompetence and a reversal of the left atrioventricular diastolic pressure gradient. Clearly in these cases forward flow through the mitral valve in late diastole could not play any part in the production of the murmur. The next year H. E. Aldridge and his colleagues ${ }^{6}$ demonstrated diastolic $^{2}$ mitral insufficiency by cineangiography in nine patients with severe aortic incompetence and reversed pressure gradients. It remained for S. Lochaya, M. Igarashi, and A. B. Shaffer ${ }^{7}$ to suggest that the Austin Flint murmur commonly found in severe aortic incompetence was due to diastolic mitral incompetence. They reported three cases with cineangiographic evidence of late diastolic mitral regurgitation coinciding with an Austin Flint murmur recorded in the phonocardiogram.

Given that diastolic mitral regurgitation does indeed sometimes occur in severe cases of aortic incompetence, two further problems remain. What causes the mitral valve to become incompetent in late diastole? And are the vibrations set up by this regurgitant puff responsible for the Austin Flint murmur ? Lochaya and his colleagues ${ }^{7}$ suggest that dilatation of the mitral valve ring due to left ventricular enlargement may result in incomplete closure of the mitral orifice by the valve cusps. The normal function of the chordae tendineae might be impaired by the great ventricular dilatation, and another factor could be partial displacement of the closed or nearly closed mitral valve cusps during the atrial systole. Whether a small puff of mitral regurgitation would be sufficient to produce a loud mitral diastolic murmur is uncertain, but it is difficult to present a satisfactory alternative explanation for the murmur in patients with a reversed diastolic atrioventricular gradient.

So the Austin Flint murmur may have a different pathogenesis in individual cases of aortic incompetence, depending on the direction of the left atrioventricular diastolic pressure gradient. When the murmur occurs with less severe aortic incompetence and without reversal of the left atrioventricular

\footnotetext{
Flint, A., Amer. f. Med., Sci., 1862, 44, 29.

- Wright, J. L., Toscano-Barboza, E., and Brandenburg, R. O., Proc. Mayo Clin., 1956, 31, 120.

Welch, G. H., jun., Braunwald, E., and Sarnoff, S. J., Circulat. Res., $1957,5,546$.

- Dodge, H. T., Sandler, H., and Evans, T., Circulation, 1960, 22, 741.

- Wigle, E. D., and Labrosse, C. J., ibid., 1965, 32, 708.

- Aldridge, H.'E., Lansdown, E. L., and Wigle, E. D., ibid., 1966, 34, Suppl. No. 3, 42.

' Lochaya, S., Igarashi, M., and Shaffer, A. B., Amer. Heart F., 1967, 74, 161.
} 